\title{
The Westerbork Telescope and Neutral Hydrogen (HI) in the Universe
}

\section{Thijs van der Hulst}

Kapteyn Astronomical Institute, University of Groningen, Landleven 12, 9747AD Groningen, The Netherlands

E-mail: j.m.van.der.hulst@rug.nl

\section{Tom Oosterloo}

ASTRON

Oude Hoogeveensedijk 4, 7991 PD Dwingeloo, The Netherlands

E-mail: oosterloo@astron.nl

\section{Raffaella Morganti}

ASTRON

Oude Hoogeveensedijk 4, 7991 PD Dwingeloo, The Netherlands

E-mail: morganti@astron.nl

50 Years Westerbork Radio Observatory, A Continuing Journey to Discoveries and Innovations Richard Strom, Arnold van Ardenne, Steve Torchinsky (eds) 


\section{Chapter 12 The Westerbork Telescope and Neutral Hydrogen (HI) in the Universe}

Thijs van der Hulst', Tom Oosterloo², Raffaella Morganti²

I n 1944 Henk van de Hulst made the pioneering discovery that radiation from neutral hydrogen atoms in the universe is observable at a wavelength of $21.11 \mathrm{~cm}$, i.e. with a radio telescope. This discovery fundamentally changed astronomy in the Netherlands. After the important early work with the Kootwijk and Dwingeloo telescopes, focused on mapping the HI in our own Galaxy, the Milky Way, the WSRT followed this lead and showed that not only the Milky Way and its neighbour the Andromeda galaxy, but the majority of the galaxies in the universe are full of neutral hydrogen with similar distributions and kinematics. Neutral hydrogen (HI) observations throughout the following decades has taught astronomers a lot about the structure and evolution of galaxies from observing the HI. Work with the WSRT has been world-leading from the start and, because of a series of improvements of the telescope over the years, is continuing to play an important role in the field of extragalactic $\mathrm{HI}$ astronomy.

The WSRT was originally designed for measuring wideband radio radiation from radio-galaxies and quasars and from star formation in galaxies. Thanks to a clever modification of the system, engineers and astronomers made the WSRT suitable for measuring the $21-\mathrm{cm}$ line radiation of neutral hydrogen in the early seventies. It was important to do this at the time because it gave the WSRT a unique advantage over other telescopes. Around 1980 the VLA (Very Large Array in New Mexico, USA) would be put into operation. Having spectral line capabilities before this date gave the WSRT a unique position regarding kinematical studies of nearby galaxies. Later also the VLA obtained spectral line capabilities, but the WSRT stayed ahead again by deploying very stable, broadband correlators, first the DXB and then the IVC.

The WSRT hardware had 80 analog channels, which were used for broadband work and allowed for measuring the 80 signals from all four possible combi-

1 University of Groningen, The Netherlands 2 ASTRON, University of Groningen, The Netherlands nations of the two receivers per telescope for 20 baselines (telescope pairs). The change to the existing hardware was a modification to these 80 channels to simultaneously measure the signal of 10 baselines in 8 narrowband filters $(27 \mathrm{~km} / \mathrm{s}$ wide and separated by $40 \mathrm{~km} / \mathrm{s}$ ) instead of 40 broadband signals from 20 baselines. A full sampling of a spectrum with 20 baselines required 

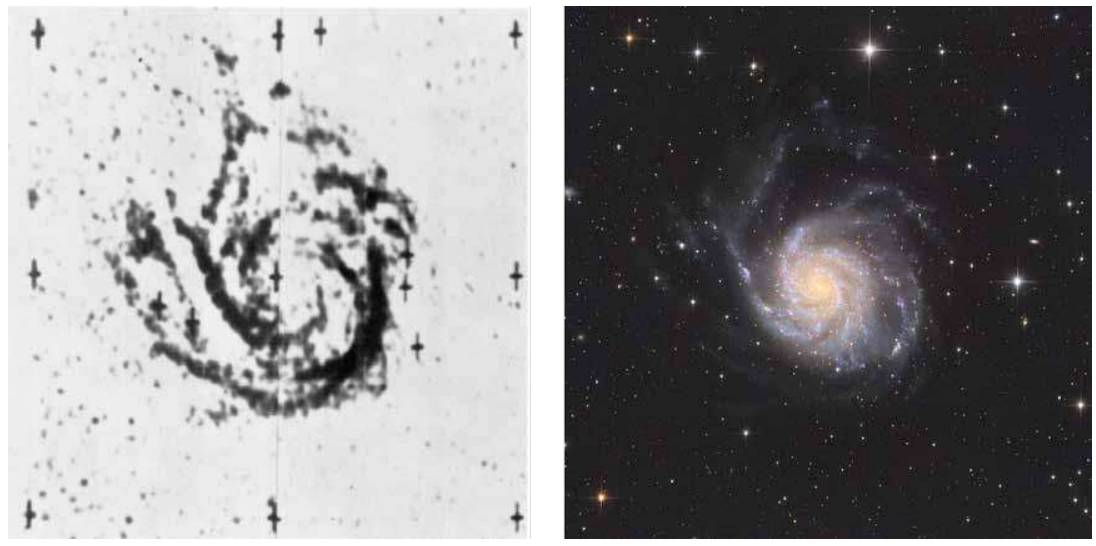

4 measurements in practice. Slow compared to the broadband work, but sufficient for establishing a number of scientific breakthroughs. This was possible because, despite the long observing time for a fully sampled observation with the WSRT, there was actually no competition. The sensitivity and spatial resolution of the WSRT were much better than any other interferometer at the time.

With this 8o-channel receiver, it was possible for the first time to map in detail the structure and kinematics of the neutral hydrogen gas in galaxies and this actually opened up a whole new area of research. Because of the unique capabilities offered by the WSRT, each observation immediately yielded new and interesting results. What became clear first was that the neutral hydrogen in spiral galaxies shows the same kind of spiral structure as thought to be present in the Milky Way and that the distribution of the gas follows in particular that of the bright young stars. Figure 1 shows the first Westerbork image of the neutral hydrogen (HI) distribution in the large nearby spiral galaxy Messier 101. This clearly demonstrated that the $\mathrm{HI}$ in galaxies also exhibits spiral structure. The early HI observations also showed that the gas disks of galaxies are larger than the stellar disks (on average one and a half times) and that the outer parts of the gas disk often have a different orientation, the so-called 'warps'. One of the largest warps to date has been found in the galaxy NGC 4013 and is shown in figure 2. The precise origin of such warps is still under investigation. The main cause is thought to be external, either a steady inflow of fresh gas or the capture of small satellite galaxies.

The most important work that has been carried out with the 80-channel receiver is undoubtedly the use of neutral hydrogen to measure the rotation of galaxies at much larger distances from their centres then possible optically, and hence derive from the rotation the total mass distribution in spiral galaxies out to these large radii. Astronomers use the Doppler effect to measure the velocity of the neutral hydrogen along the line of sight, and in this way the rotation of the gas disk around the centre of a galaxy can be measured. This measurement can be used to estimate the total mass, just as the mass of the sun can be de-
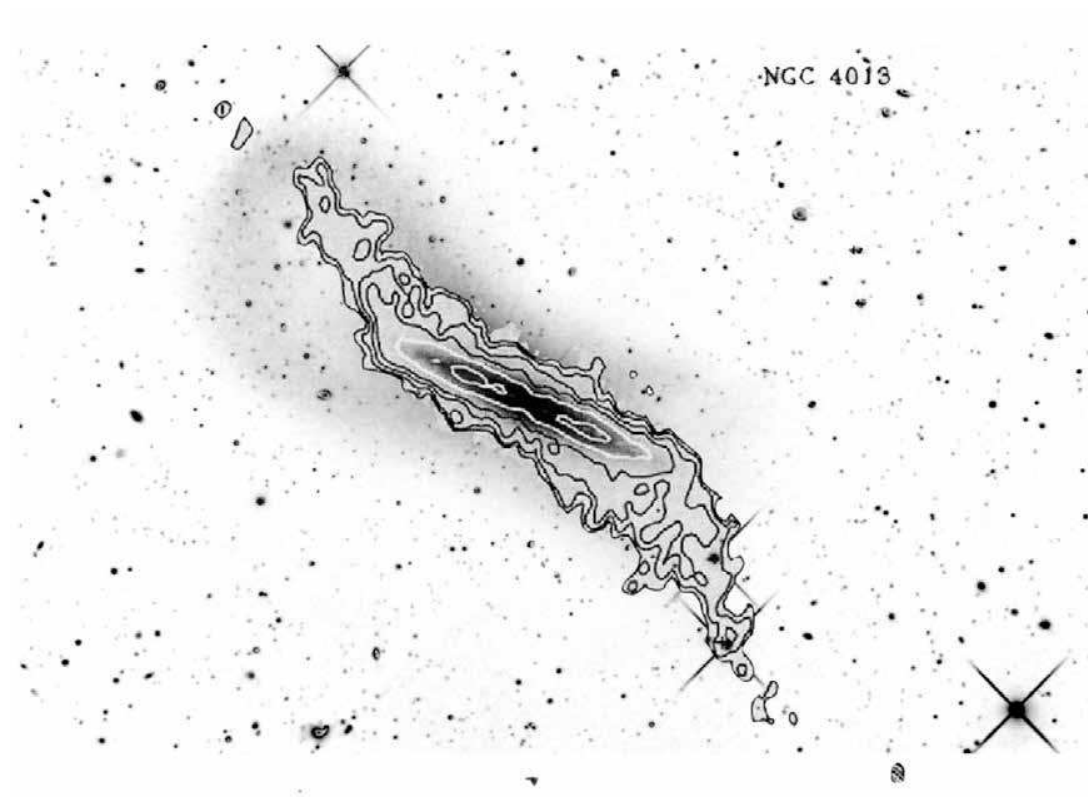

termined from the orbital speed of the earth and the other planets around the sun. The orbital speed is a measure of the mass: the mass, that is to say gravity, pulls the gas clouds inwards but a sufficiently high orbital speed prevents this from happening. The larger the mass, the faster the gas must move so as not to fall to the centre. So in other words, if we know the speed and the distance to the centre we can determine the mass within that radius.

Bosma first showed (PhD thesis 1978, and Astronomical Journal 86, 1825 \& 1981 ) that the majority of galaxies show flat rotation curves out to the largest measured radii, indicating that the total mass keeps increasing with radius, other than the distribution of light in galaxies suggest. This is illustrated in Figure 3. This led to the notion of the so-called 'dark matter', as the rotation indicated that there must be more matter present than is observed in the form of gas and stars. This 'dark matter', whose nature has not yet been determined up to this day, plays a fundamental role in modern astronomy and cosmology. In the 1980 s, opinions were divided on how exactly the dark matter in galaxies was distributed. "How much is there?" and "where exactly is it?" were important and unanswered questions. Because the neutral hydrogen in galaxies extends much farther than the observable distribution of stars, the WSRT measurements of the movements of the gas in galaxies yielded the best determinations of the mass distribution in these systems. It became clear that there is significantly more matter in galaxies than the sum of all stars, dust and gas and that most of the dark matter resides in the outer parts of galaxies. This led to more detailed modelling of the rotation of galaxies taking into account both the visible and the dark matter. Of these studies the one of NGC 3198 has become the classical example and is shown in Figure 4. 

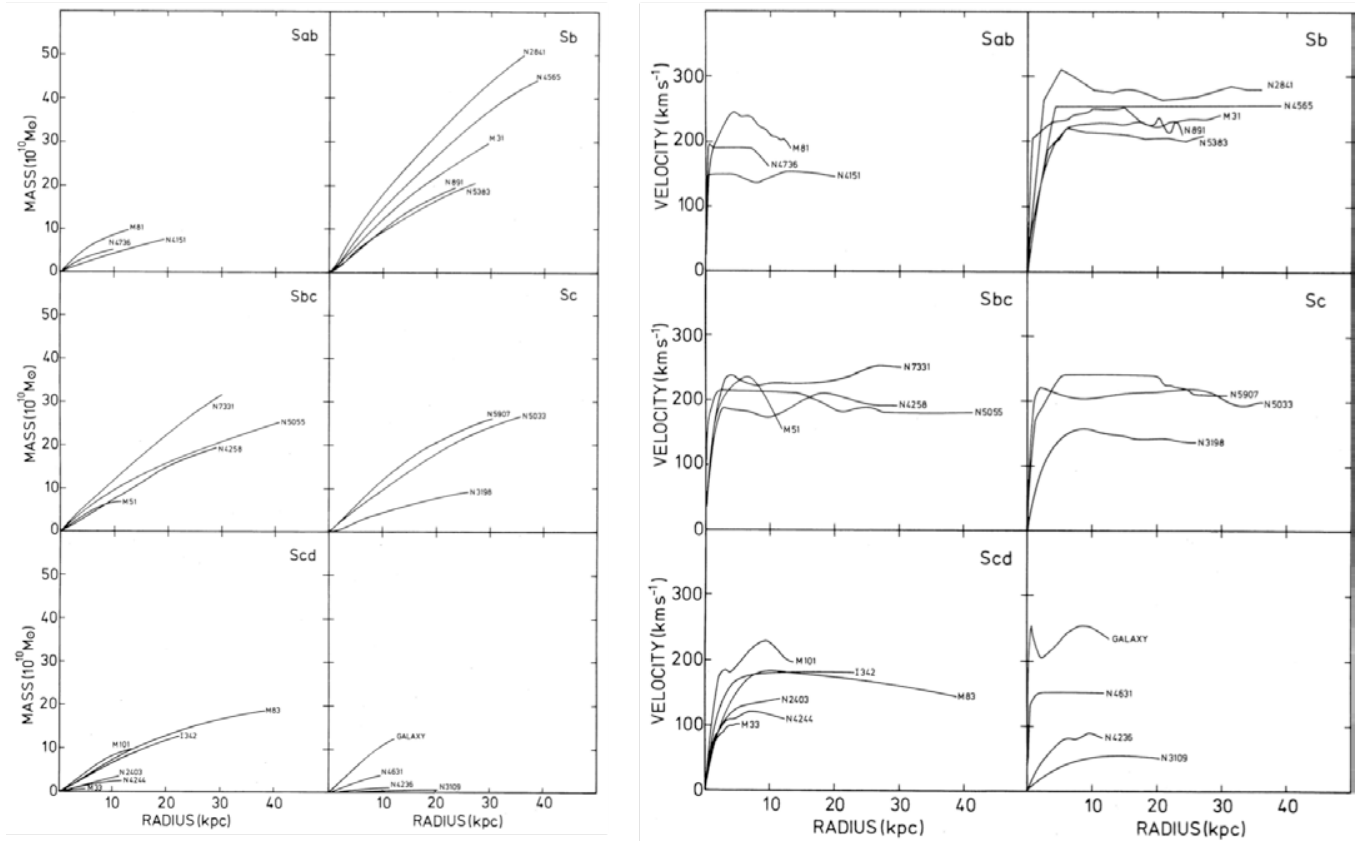

Figure 3: Rotation curves (right panel) and cumulative total mass distributions of 25 galaxies studied by Bosma (Astronom cal Journal, 86, 1825, 1981). It is clearly demonstrated that extended rotation curves remain flat and that the corresponding cumulative total masses continue to increase and do not converge to a constant value.

Figure 4: The

extended and flat $\mathrm{HI}$ rotation curve of the spiral galaxy NCC 3198, illustrating the discrepancy between the measured rotation and the rotation and the
rotation expected on the basis of the measurable mass in the disk, requiring a dark matter halo component (van Albada, Bahcall, Begeman at Sancisisi Astrophysical Jour-

nal 295, 305, 1985)
DISTRIBUTION OF DARK MATTER IN NGC 3198

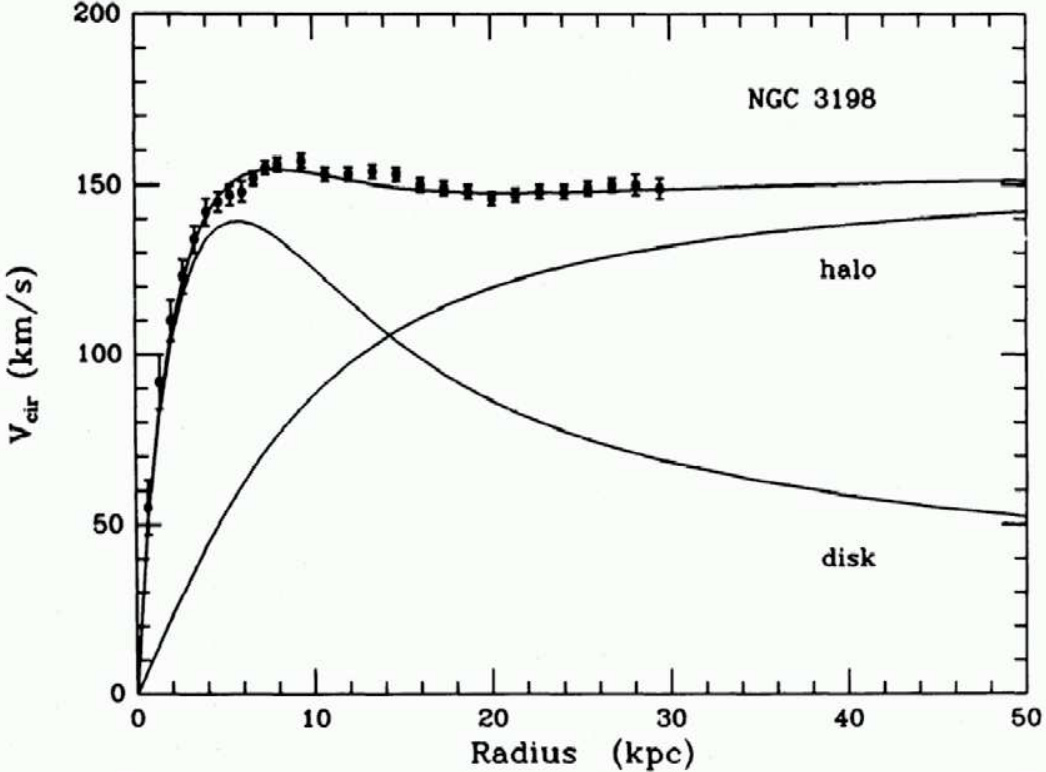

Another nearby galaxy observed in detail in $\mathrm{HI}$ in those early days is Messier 81 , a spiral galaxy with three close companion galaxies Messier 82 NGC 3077 and NGC 2976. The studies of Messier 81 showed not only the complexity of rotation curves (its rotation curve is asymmetric) but also the presence of strong non-circular motions in the gas orbits around the spira arms, caused by so-called density waves, and it became the first galaxy in which the density wave theory was properly tested by Visser (Astron. \& Astrophys. 88, 149/159, 1980, Bash \& Visser, Astrophysical Journal 247, 488, 1981). In addition, it appeared that there is a lot of filamentary HI in between Messier 8 and its companion galaxies, clear signs of the effects of gravitational interaction (like the ocean tides on the earth caused by the presence of the moon) between Messier 81 and its satellite galaxies. We now know that such HI structures exist in many groups and can be used to probe whether galaxies in a group have strong tidal interaction or not. Figure 5 shows Messier 81 and the first images of the intergalactic HI.

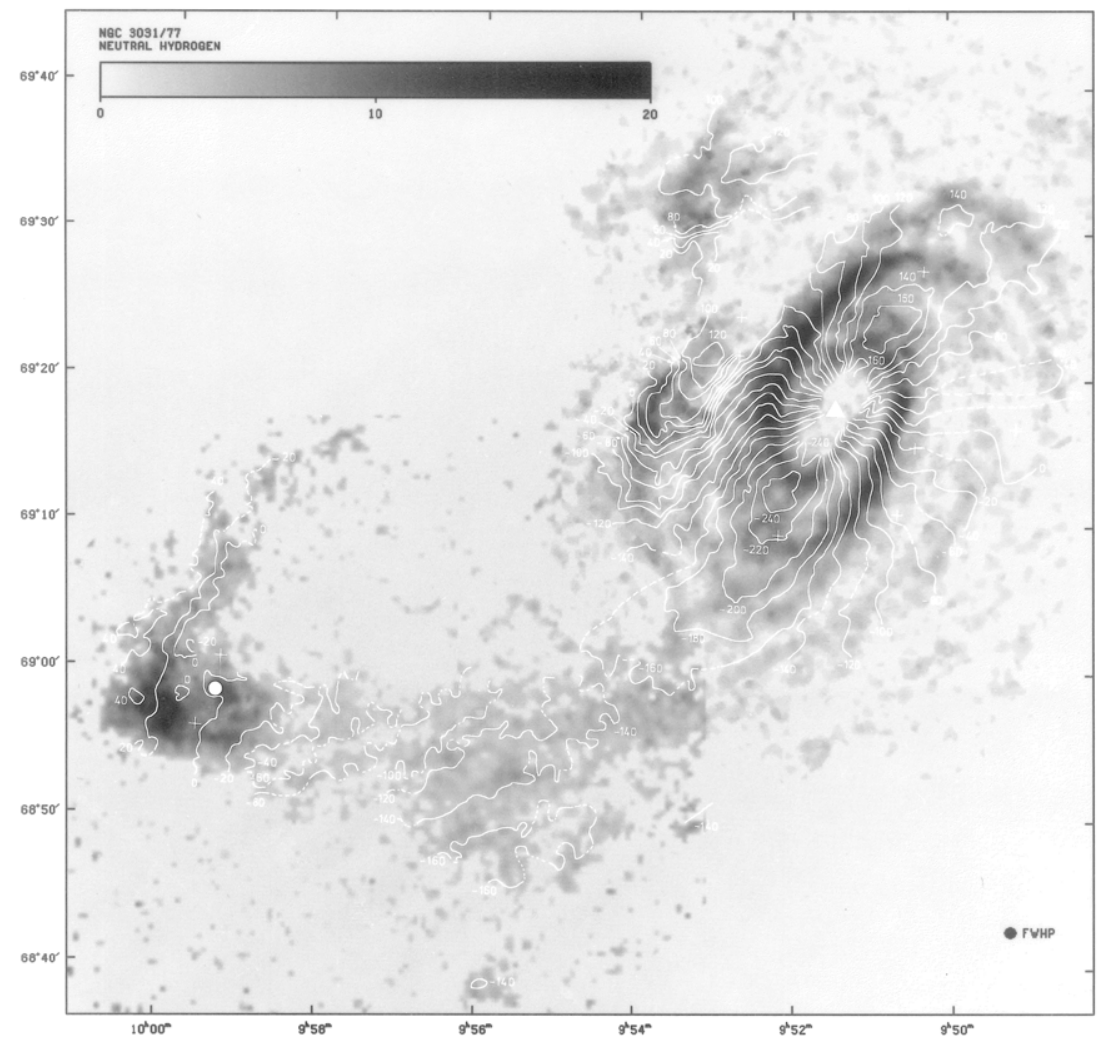

Figure 5: Early HI observations of Messier 81 and NGC 3077 showing both the streaming motions in the isovelocty contours crossing the spiral arms of Messier 81 and the Hl bridge and other intergalaxtic HI features between Messier 81 and NCC 3077 to the lower left tuan der Hulst,

Astron. \& Astrophys.

75. 97, 1979). The white triangle and

white dot indicate

the optical centres of

Messier 81 and NCC

3077 respectively.

Roughly every 10 years a significant improvement in the capabilities of the WSRT has led to new breakthroughs. The first was the construction, in the late seventies, of a digital correlator that could handle significantly more than 80 channels: no less than 5120 . This increased the observing speed for emission 
by neutral hydrogen by a factor of four and also enabled a single observation to cover a larger range in velocity coupled to a much higher velocity resolution than before. This made it possible to observe not only the hydrogen in large groups and clusters of galaxies, but also to detect the neutral hydrogen absorption of strong continuum radiation in radio galaxies and active galactic nuclei (so-called AGN) over a wider range of velocities. At the same time, the WSRT was expanded with two more telescopes, placed $1.5 \mathrm{~km}$ eastward of the existing array, thereby increasing the angular resolution of the radio images by

Figure 6: A selection of PhD theses that have used WSRT rotation curves, dark matter and the Tully-Fisher relation in galaxies. a factor of two. This was of great importance to a wide range of research areas, including $\mathrm{HI}$ research. The number of galaxies for which $\mathrm{HI}$ observations were available increased from about a dozen to several tens, slowly enhancing our knowledge of the distribution of $\mathrm{HI}$ and via the rotation curves the distribution of all matter in many types of galaxies. Figure 6 gives a quick overview of theses that have as a main component the study of rotation curves and dark matter in galaxies.

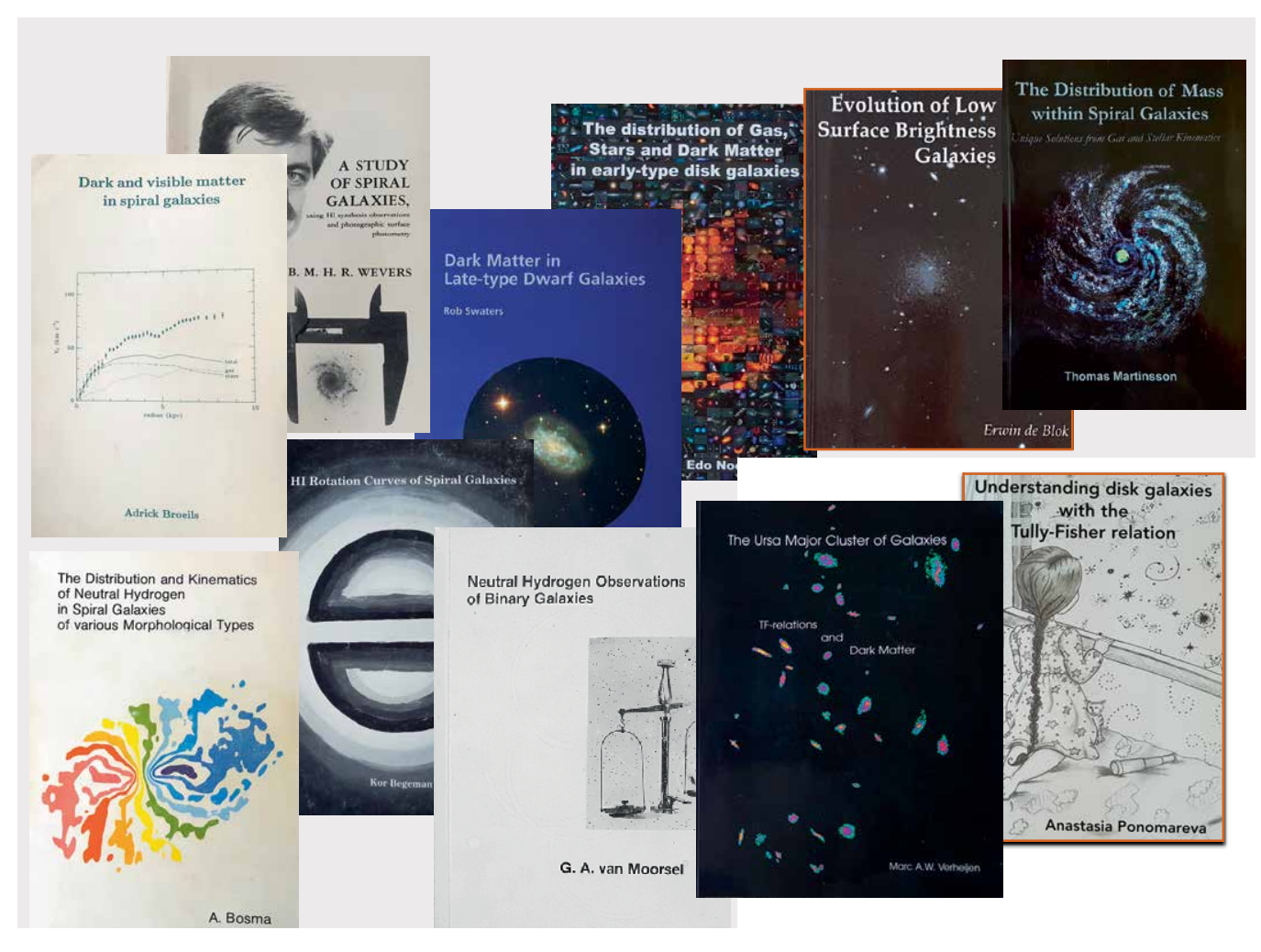

In the period 1992 to 2002 an ambitious observing program started entitled WHISP (the Westerbork HI Survey of SPiral galaxies, van der Hulst, van Albada \& Sancisi, 2001 ASP Conf. Proc. 240, 451). The aim was to observe 1000 galaxies in the nearby universe, covering a wide range of galaxy types and luminosities with as its main goal to study the systematics of the dark matter (and the Tully-Fisher relation) in galaxies of different type and mass and in different environments. When the WHISP program ended just after the turn of the century some 290 fields had been observed. The data were used for a number of theses on dark matter in galaxies, warps in galaxies, and to date the data are still widely used for many different projects, ranging from studying the relation between star formation and the gas density in galaxies to studies of the HI diameters in galaxies. The WHISP data continue to be an important resource until in a few years the blind surveys with Apertif and other instruments will have produced similar data for many more galaxies.

Halfway through its long observing period the WHISP project profited from a third major upgrade of the WSRT, the installation of the so-called multi-frequency-front-ends (MFFEs). For $21 \mathrm{~cm}$ line work these brought a significant increase in sensitivity as with the MFFEs not only the movable telescopes had cooled receivers, but also the ten fixed telescopes were now equipped with $30 \mathrm{~K}$ systems, increasing the sensitivity by a factor two. In addition the first digital correlator was replaced by a new system that has no less than a quarter of a million channels and is also equipped with adequate analogue hardware that can measure a total bandwidth of $160 \mathrm{MHz}$. This corresponds to a speed range of about $32000 \mathrm{~km} / \mathrm{s}$, or one can measure galaxies at distances between a few thousand and more than a billion light-years away. Detecting the weak hydrogen signal in such distant systems requires long observations to acquire sufficient sensitivity. This has been done for a small number of select areas.

Though WHISP had mostly focused on gas rich galaxies (and therefore mostly spiral and irregular galaxies) this now opened the way for observing gas poor systems, such as the elliptical and So galaxies, much more effectively. This led to the successful programs of first observing a modest sample of such galaxies taken from the SAURON project and then to the much larger program called Altas3 D, described below.

In 2004 the WSRT Programme Committee decided to allocate a considerable amount of observing time to observe neutral hydrogen in a large sample of early-type galaxies, until then believed to be depleted of gas. The increased sensitivity and capabilities of the (then) new MFFE receivers offered an ideal opportunity to begin studying this class of galaxies in detail. So called early-type galaxies, in particular the elliptical galaxies are different from spiral galaxies in that their light distributions are smooth, do not form new stars and, therefore, consist mostly of old stars. Therefore they are often referred to as red-and-deadl Optical images of spiral galaxies on the other hand clearly show that they consist of a mix of stars, gas and dust and that many new stars are continuously formed. The reason for this difference is thought to be the lack of gas in early-type galaxies, inhibiting the production of stars. Early work with the WSRT (e.g. van Driel \& van Woerden, 1991 Astron \& Astrophys. 243, 71) showed that some early-type galaxies do have gas, except that the densities of the gas are too low for star formation. Obtaining a complete view of the gas content of 
early-type galaxies was, however, limited by the sensitivity of the available radio telescopes until the MFFE upgrade of the WSRT.

The first group of elliptical galaxies for which deep HI observations were obtained, was the so-called SAURON sample, earlier studied in detail using the innovative optical integral field spectrograph SAURON on the William Herschel Telescope (WHT), providing the complete view of the stellar component and of the ionised gas. The deep HI observations from the WSRT complemented this and showed that the picture regarding gas in early-type galaxies is much more complicated than expected.

The first surprise was the high detection rate: HI was detected in about a quarter of galaxies, a much higher fraction than expected. Another surprise was that in many cases the HI was distributed in a regular disk. Figure 7 shows NGC 4278 , where a regular HI disk can be seen, twice as large as the optical body of the galaxy. Like in NGC 4278 , the majority of the cases show a strong similarity between the kinematics of the $\mathrm{HI}$ and that of the ionised gas, suggesting a similar origin of the gas, while the gas kinematics can be quite different from that of the stars (as in Figure 7). In some cases, the gas is even counter-rotating to the stars suggesting that the gas has an external origin.

Figure 7: Images representing the motion of gas and stars in the galaxy NCC4278. The red regions are moving away from us, the blue are approaching us. The foure represents a nice example of the complementarity of the data. On the left the neutral hydrogen as measured by the WSRT, on the right the ionized gas (bottom) and stars (top) as measured by the integral field spectrograph SAURON on the William Herschel Telescope on Palma Note the different scales that La two instruments con imege: the SAURON data cover the ve of the Hl image (black box).

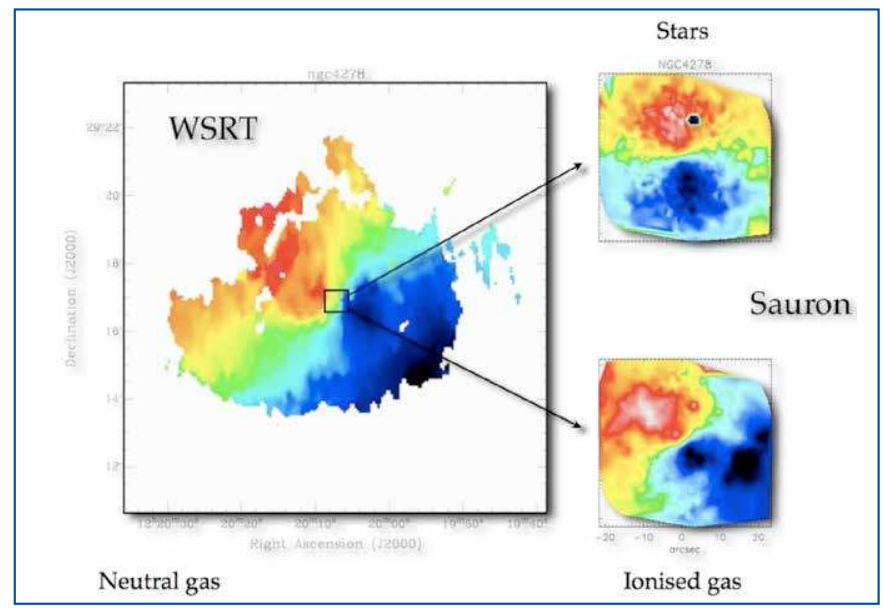

Figure 8 shows NGC 4203, a good example of the (huge) extent of the HI disks found in some of the early-type galaxies. The amount of HI in early-type galaxies is often similar to what is found in spirals, but because of the huge extent, the typical surface density of the gas is much lower, too low to form stars. This illustrated that the difference between early-type galaxies and spirals is not so much that early-type galaxies do not have gas, but that the density of the gas is too low for active star formation. The results of this study have been presented in Morganti et al. Monthly Notices RAS 371, 157, 2006 and Oosterloo et al. Monthly Notices RAS 409, 500, 2010.

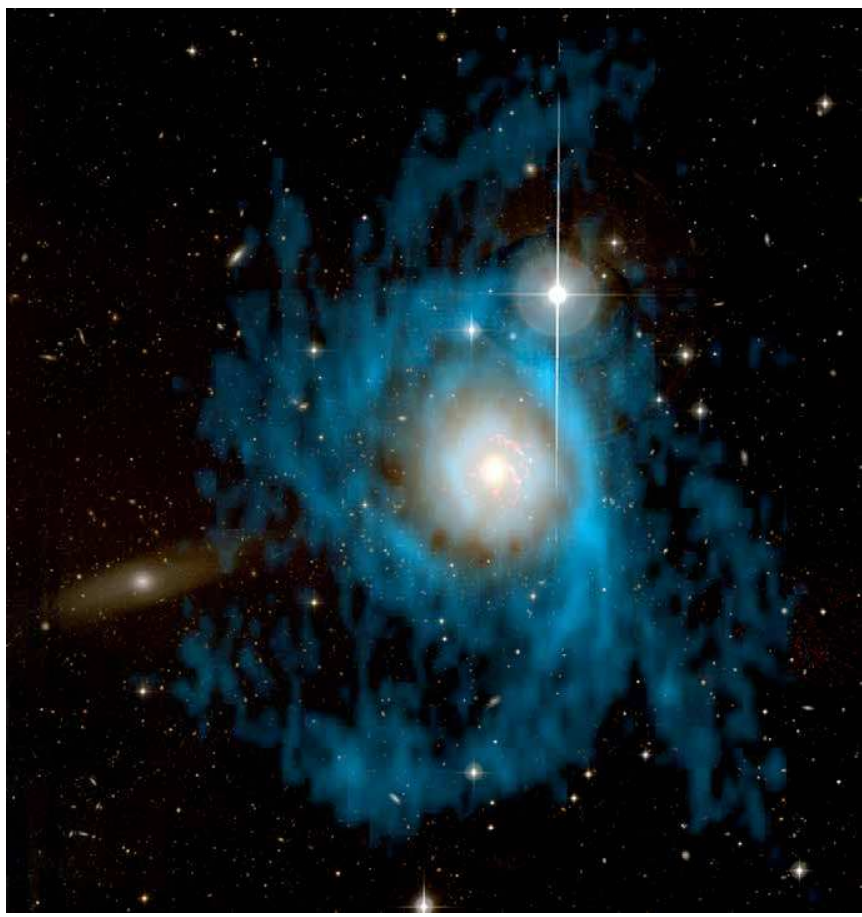

Figure 8: HI emission in $\mathrm{NCC}_{42} \mathrm{O} 3$ (blue in the image) observed by the WSRT (Yildiz, M.K et al. 2015, Monthly Notices RAS, 451,103). They find that the gas system (shown in blue) consists of two sep. arate components: an inner regularly rotating ring and an outer irregular HI structure which likely has an external origin

This study led to a larger project aimed at observing more than 100 early-type galaxies part of a volume-limited, complete sample: the Atlasd3D survey (Cappellari et al. 2011 Monthly Notices RAS 413, 813). The WSRT observation of this much larger sample (Serra et al. 2012 Monthly Notices RAS 422, 1835) not only confirmed the previous results, but also showed that the distribution of the $\mathrm{HI}$ (regular disks versus compact disks and HI tails) depends strongly on the environment of the galaxy. This is demonstrated in Figure 8. Early-type galaxies in poor environments (top row in Figure 9) typically host giant HI disks with radii up to many tens of kpc. The disks are very regular, indicating that the host galaxy has been undisturbed for a very long time.

Figure 9: Examples of $\mathrm{Hl}$ in early-type galaxies in different environments (Seria et al. Monthly (835, 2012) 1835, 2012)

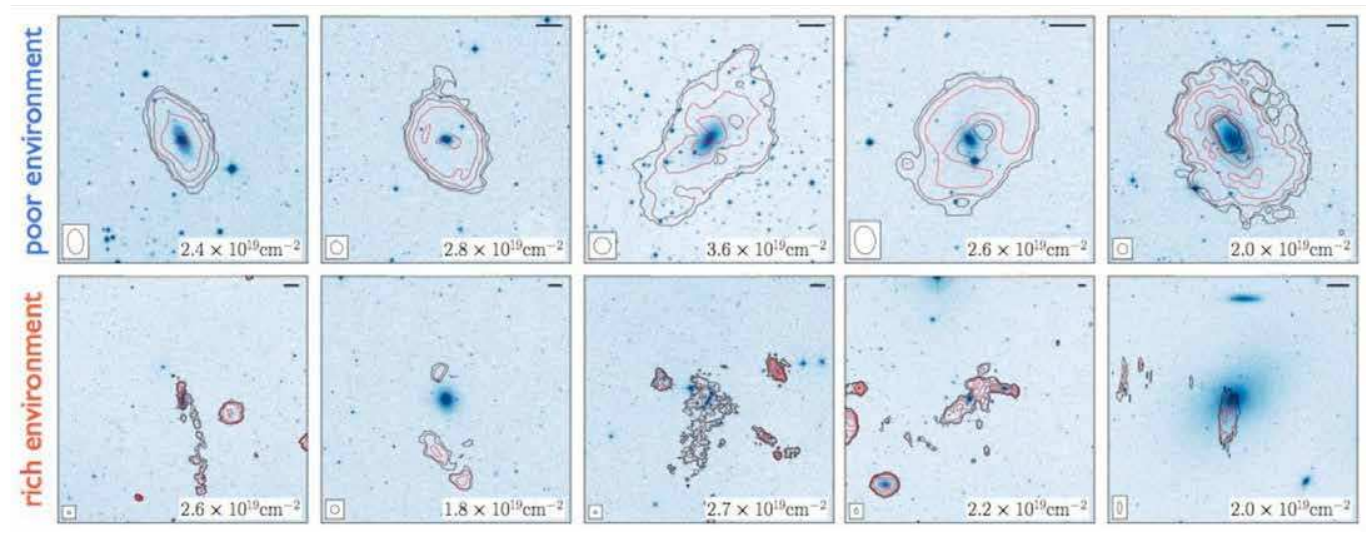


The situation is very different in richer environments like galaxy groups and at the outskirts of galaxy clusters (bottom row in Figure 9). Here the HI typically has a very disturbed morphology. In many cases long HI tails stretch from the host galaxy into the surrounding space, demonstrating that some gas may have recently been removed from (or accreted onto) the galaxy. Early-type galaxies in these environments are evolving because of the interaction with what is around them. At even denser environment densities, in the very centre of clusters, hardly any $\mathrm{HI}$ is found in galaxies. These galaxies have moved through the hot cluster medium and were stripped from their $\mathrm{HI}$ on their way to the cluster centre where the intra-cluster medium is most dense.

The elliptical galaxies described above have been favourite targets for another reason. The super-massive black holes (SMBH) hosted in their centres can emit spectacular collimated radio jets shining bright at radio wavelengths and interacting with the surrounding medium. Because of the strong radio continuum emission it becomes possible to study the gas in these very central regions because it reveals its presence by absorbing the continuum emission. This allows us to trace the presence of even small amounts of gas: The stronger the radio source, the easier it is to detect even a small HI cloud. The initial studies of HI absorption (also done with the WSRT) focused on tracing HI gas with velocities within a few hundred $\mathrm{km} / \mathrm{s}$ of the systemic velocity of the host galaxy, i.e. looking for gas from circumnuclear disks or similar structures around the SMBH.

The expansion of the WSRT backend to broader bandwidth (with the IVC) offered the unique opportunity of tracing the HI gas over a much broader range of velocities. This technical capability led to a major surprise when in $2003 \mathrm{HI}$ absorption observations of the nuclear region of the radio galaxy $3 \mathrm{C} 293$ showed that, in addition to a narrow absorption component associated with a circumnuclear HI structure, a very broad, blueshifted and very shallow wing of absorption was detected, extending to velocities more than 1000 $\mathrm{km} / \mathrm{s}$ lower than the systemic velocity. Figure 10 shows a cartoon illustrating the HI absorption profile and the location of the narrow and the broad velocity component. This has now been seen in a number of other AGN (e.g. Morganti et al. 2005 Astron. \& Astrophys. 444, L9). The first inventory of how common these HI outflows are, has also been done with the WSRT. A large HI absorption survey, comprising observations of more than 250 radio galaxies, has been done in preparation for larger surveys with Apertif, the new Phased Array System on the WSRT). This was, in fact, the last survey of the old Westerbork Synthesis Radio Telescope. The project (see Maccagni et al. 2017, Astron. \& Astrophys. 604, 43) went on until the WSRT was gradually refurbished for the installation of the Apertif 'Phased Array Feeds' (PAFs) on the WSRT.

In some radio galaxies, the $\mathrm{HI}$ outflow has been further studied at higher spatial resolution using Very Long Baseline Interferometry reaching even parsec scales (Morganti et al. 2013, Science 341, 1082; Schulz et al. 2018). These obser-

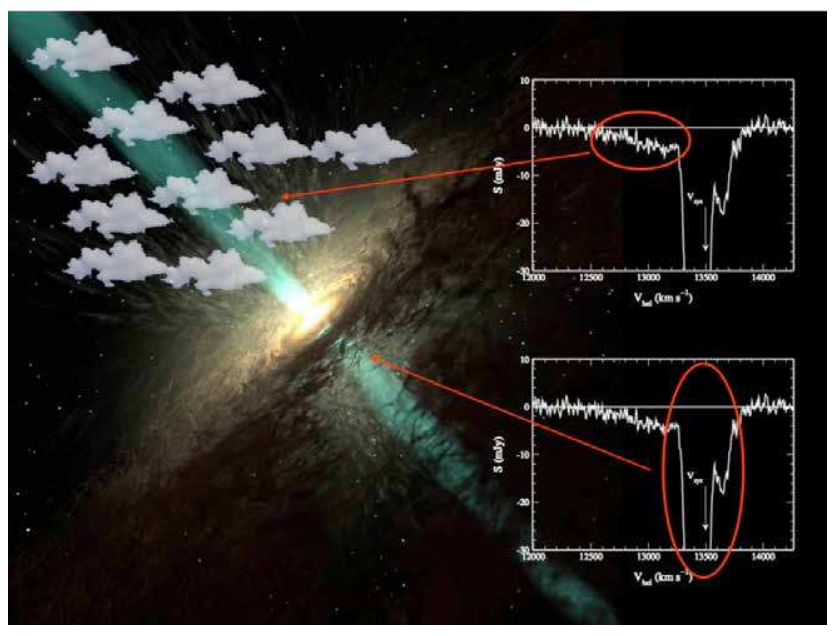

Figure 10: Cartoo taken from Morganti a Oosterloo 2018, Astron. Q Astrophys. Rev.) showing the HI ab. sorption observed in 3C293 obtained with the WSRT (Morgantiet al. 2003 Astron. Q Astrophys. 593, $L 69)$ and the components of gas that can produce the various parts of the absorption profile.

vations have given direct proof that, at least in some cases, the outflowing gas is accelerated by the interaction with the expanding radio jets. The data have shown that the outflows appear to be relatively massive, therefore likely playing a relevant role in the feedback.

In the nineties, another discovery had drawn attention: The first clear observational evidence for gas with anomalous velocities in galaxies. The most impressive high velocity complex found thus far in any galaxy is the large complex in M 101 shown in Figure 11. This discovery and the improved sensitivity of the WSRT with the MFFEs stimulated projects aimed at deep observations of a small number of galaxies in order to reveal the low column density $\mathrm{HI}$ in and around galaxies, in particular gas with anomalous velocities. This allowed hydrogen with low density to be well mapped and has led to a number

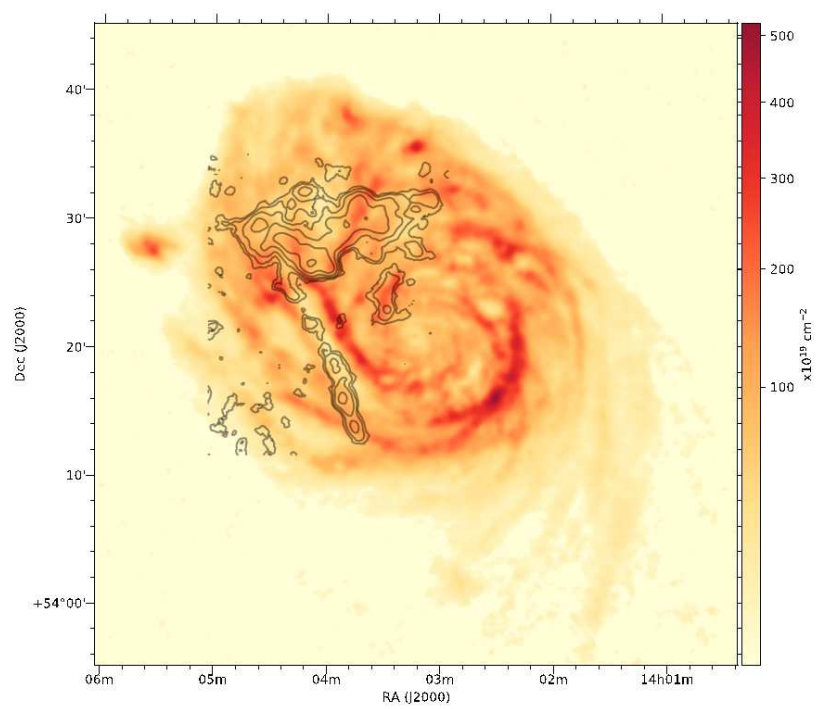

Figure 11: The high velocity gas complex in Messier 101 shown in black contours superposed on the HI column density disTribution in orange. The image has been made from recent W RT observations of Messier 101 by Oosterloo et al. 
of interesting insights that are directly related to the influx of fresh gas from the surrounding area as well as the redistribution of gas in a galaxy as an ultimate result of the formation of especially massive stars. They produce powerful stellar winds at the end of their short (100 million years) existence and then explode as supernova. Both processes drive hydrogen gas out of the plane of the system and form the so-called 'Galactic Fountain'. This gas circulates above the surface for some time and then falls back under the influence of the gravity of the disc. This concerns a small part of the total hydrogen in a system, but this process has now been convincingly demonstrated on the basis of sensitive measurements. All this has led to an image in which galaxies are constantly in a dynamic balance in terms of their gas disk: a part of the gas leads to the formation of stars, these drive out and in turn displace part of the gas and in addition 'fresh' gas into the galaxy from the intergalactic space.

A prime example illustrating such phenomena is the edge-on galaxy NGC 891, shown in Figure 12. Its gas disk is clearly 'fatter' than the stellar disk and in addition it shows long filaments that witness the infall of fresh gas into the galaxy. NGC 891 is a remarkable object. Its hydrogen distribution was observed many times with the WSRT throughout the fifty years of its existence and rather than becoming more extended radially the increased sensitivity revealed this galaxy to get 'fatter' and 'fatter' in $\mathrm{HI}$ and revealed the vertical distribution of $\mathrm{HI}$ to very low levels, also uncovering the $\sim 20 \mathrm{kpc}$ long filaments in the northwest. These observations showed that the bulk of the gas above and below the disk is corotating with the disk, though at a somewhat lower velocity. There is no information on the velocities perpendicular to the disk because NGC 891 is seen edge-on and the Doppler motion only measures velocities along the line of sight. Deep observations of the face-on galaxy NGC 6946 (Boomsma et al. As-

Figure 12: The neutral hydrogen distribution in the edge-on galaxy NCC 891. The contours and blue shades represent the neutral hydrogen emission. The orange figure in the center illustrates the light distribution. The neutral hydrogen data are taken from Oost erloo, Fraternali a Sancisi, Astronomi cal Journal 134, 1019, 2007). Image courte sy T.A. Oosterloo.

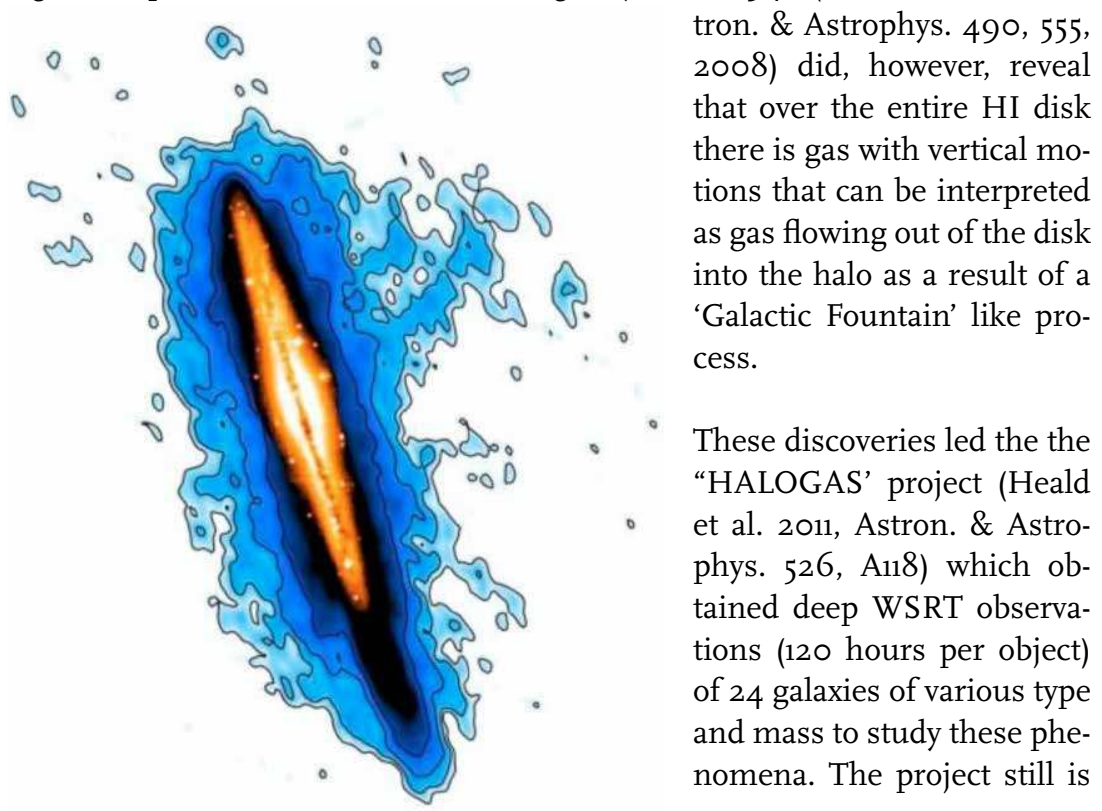

ongoing, but constitutes the most sensitive collection of high-resolution $\mathrm{HI}$ observations of galaxies for a while to come.

The increased sensitivity and bandwidth of the WSRT have greatly helped the study of HI in distant galaxies. Ultra-deep (in this case 1000 hours) observations of two distant clusters of galaxies Abell 963 and Abell $219^{2}(\mathrm{z} \sim 0.2$ or a distance of $\sim 800 \mathrm{Mpc}$ or a look-back time of $2 \times 10^{9}$ year) managed to detect more than a hundred galaxies at this distance (epoch) and allow a characterization of the HI properties of galaxies not only in the range of environments that are present around these clusters, but also at a time when the universe and hence the galaxies were of $2 \times 10^{9}$ year younger than in the local universe. Figure 13 shows three of the detections in Abell 963. These observations, combined with similar studies of clusters and groups in the nearby universe begin to show that a number of physical processes are at work: Gas stripping by an external medium, gas removal by gravitational interactions, increased start formation and its consequences (winds, gas removal) by stripping and interactions, and in addition the continuing accretion of gas from the cosmic web and re-accretion of material removed in an earlier phase. The precise balance of these processes, many of which can only be diagnosed by observing the $\mathrm{HI}$ in and around galaxies, is yet unclear and requires observations of many more objects in many different stages of their evolution and different environments. New surveys, as with Apertif (see below), will provide such information.
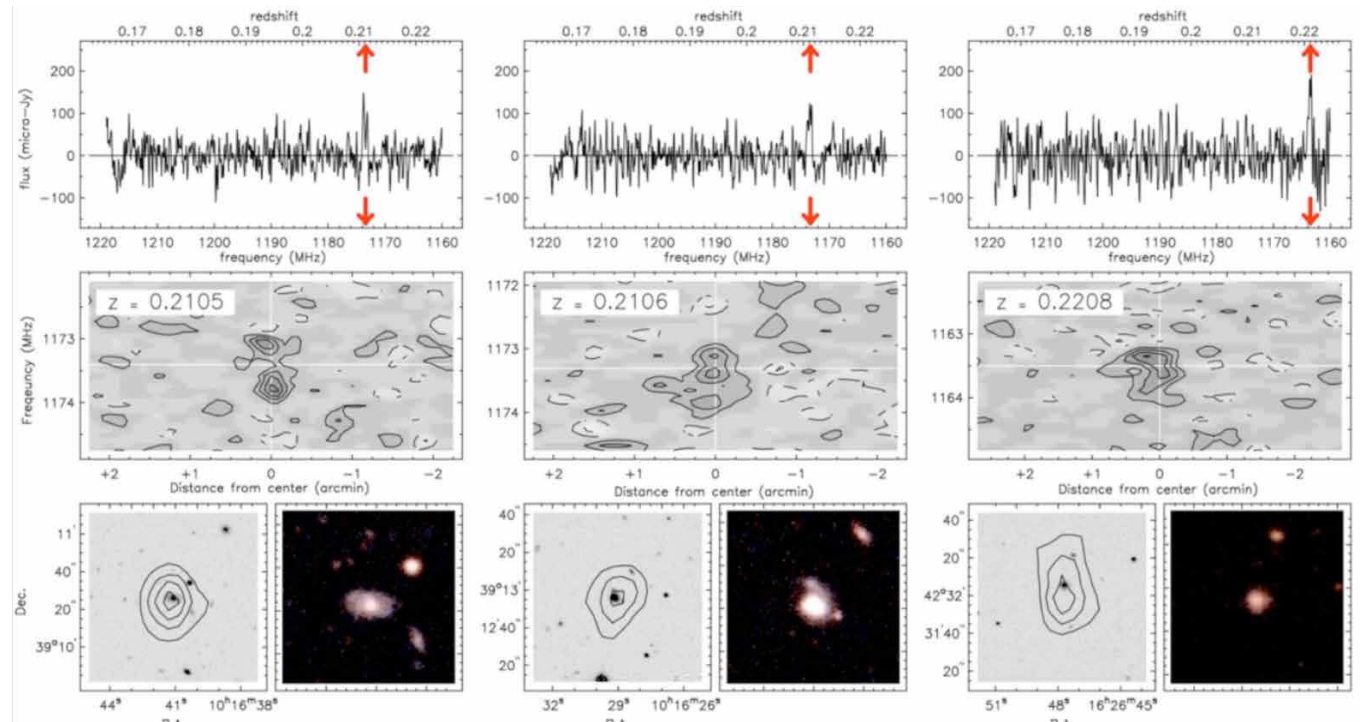

The story of what the WSRT has contributed to our understanding of HI in galaxies will be incomplete if we let the smallest galaxies and galaxies in the emptiest corners of the universe unmentioned. The most effective way to find galaxies with extremely low HI contents is with a telescope with a large collecting area, single dishes such as the Arecibo telescope, the Parkes telescope or the 


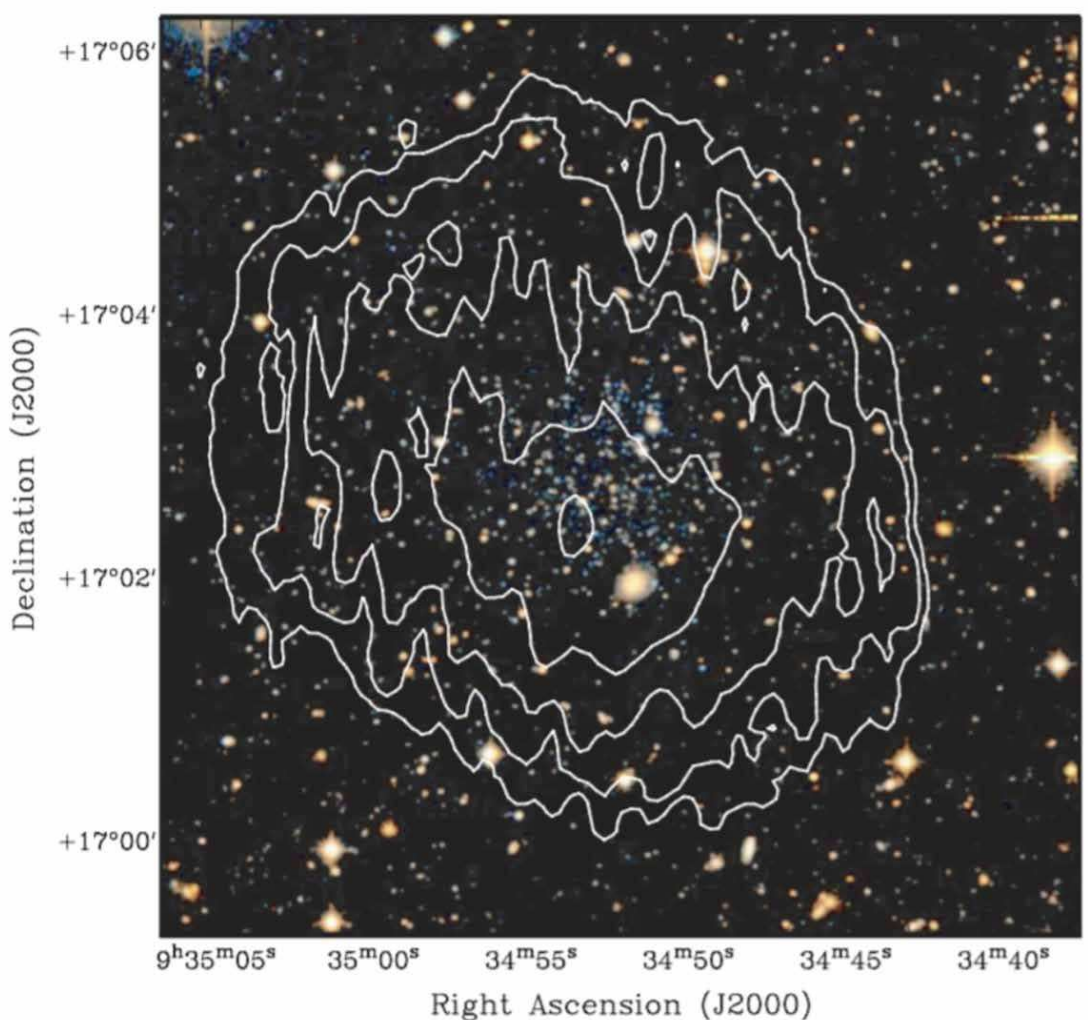

Green Bank Telescope. Surveys with the former two have indeed found objects with very low HI mass, but only follow up observations with instruments such as the WSRT have been able to confirm that the HI is in an extended, low surface brightness, rotating disk. An interesting and perhaps prototypical example, found first in the Parkes HIPASS survey and later confirmed optically and observed in detail with the WSRT, is Leo T (Figure 14), an optically insignificant galaxy, extremely rich in HI, albeit several orders of magnitude less in HI mass than typical spiral galaxies. These HI rich, dwarf galaxies have very low mass and thick HI layers, with very little rotation, though also here estimates of the total mass indicate that they are dominated by gas but also have dark matter.

The emptiest areas in the Universe are the so-called voids, huge regions, still expanding with the general expansion of the Universe, where the galaxy density (and general matter density) is at a record low. These voids are not completely devoid of galaxies and are populated by on average smallish galaxies, which do have very similar properties to their counterparts in denser environments. HI observations with the WSRT have contributed to this notion from the point of view of their HI properties. The earliest such observations were done already in the nineties, in the Boötes void, but superseded by more recent observations (carried out in 2008-2009) of galaxies in 6o carefully selected voids. Many of

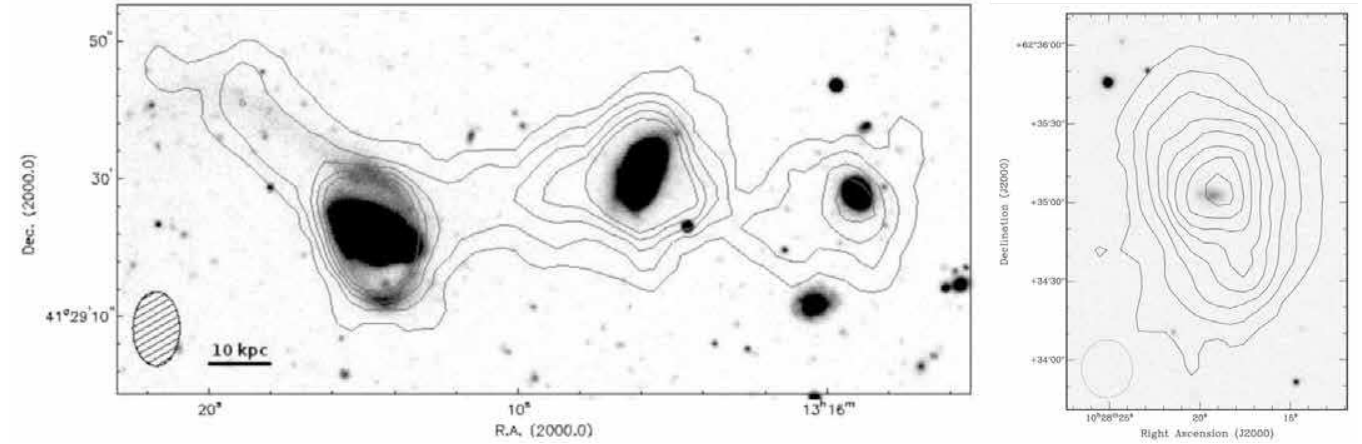

the small galaxies in voids appear to have companions, show signs of gravi- Figure 15: Two tational interaction and there may be at least two examples of galaxies in formation: The case of a galaxy with a huge polar disk which aligns well with a thin large scale structure wall between voids from which gas may have been accreted, and the case of three galaxies apparently connected by an HI structure which could be the remnant of a large scale structure filament from which these galaxies formed. Figure 15 shows these examples.

The various improvements in the WSRT system made it possible to map the hydrogen in and around a large number of galaxies, and in the last 20 years, about five hundred galaxies have been systematically observed. This does not give a complete but it's a very good overview of the properties of galaxies, both in terms of the distribution of the hydrogen in and around the systems and the distribution of the total mass on the basis of the movements of the gas. These studies form the basis for the knowledge of hydrogen in and around galaxies and are a guideline for large surveys with future radio telescopes.

The last big change for the WSRT is to equip 12 of the 14 telescopes with socalled 'phased array feeds' (PAFs). A PAF is a system of several closely spaced antennae that each sample the incoming radiation field. The signals of the elements can be combined with each other coherently to cover multiple positions in the sky. The WSRT PAF system (called Apertif, www.apertif.nl) consists of a matrix of 11 by 11 antennae that together sample 39 positions in the sky and give the WSRT effectively a 25 times larger field of view. This means that in one day an area in the sky can be measured that previously took 25 days. The realization of this technique is based on years of experience in dealing with complex radio interferometry techniques, combined with the gigantic increase in computing power of modern processors. Apertif will be ready by the end of 2018 and the WSRT will then start a large-scale survey of a significant part of the northern sky. With this, our knowledge of neutral hydrogen in and around galaxies will advance significantly, not only because we will have hydrogen images of many more galaxies, but also because a large number of different environments in the nearby universe will be mapped, both empty and densely populated areas. The latter is very important as the environment also determines which examples of galaxies in voids. The right huge hydrogen disk around a small gal. axy misaligned with the stellar body as the gas was accreted from a wall between voids. The left panel shows three galaxies, shows three galaxies,
connected by a connected by a hydrogen filament, perhaps the residual gas of the structure grom which the galaxies formed in the void. Data from
Bevgu et al. AstroBeygu et al. Astro145, 120, 2013, 
Figure 16. Recent hydrogen image of Messier 101, made with the WSRT and its sensitive MFFEs. The blue emission is the hydrogen in this galaxy, superposed on the optical image shown in orange colours. The large extent of the hydrogen as compared to the light distribution from stars and nebulae is very clear from this image. Image made from recent WSRT observations, courtesy Tom Oosterloo.

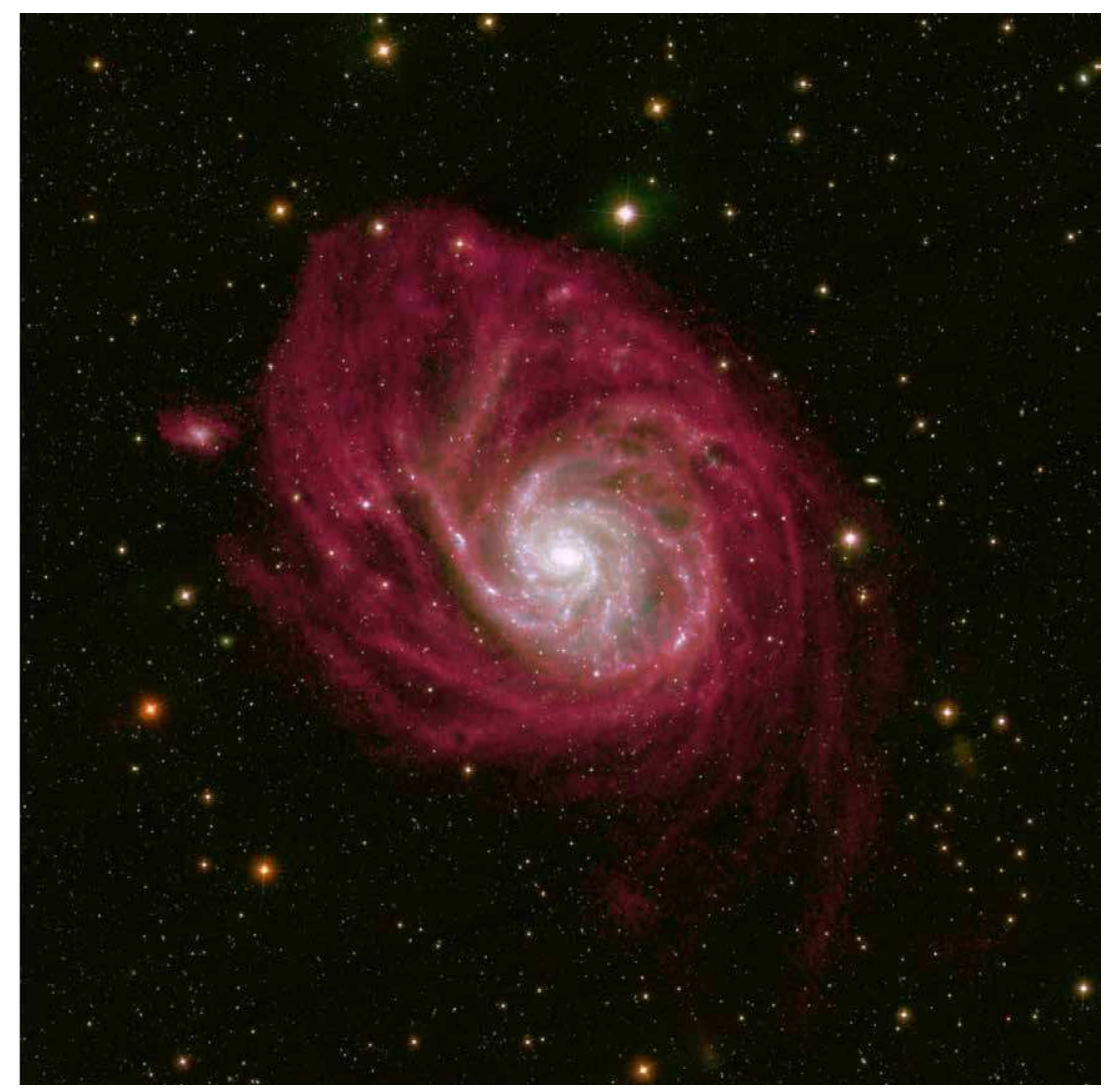

processes of gas accretion and gas removal dominate galaxy evolution, as described above. So with Apertif the WSRT will enter its fifth youth even before the respectable age of 50 is reached and will routinely be able to image large areas of sky and produce images such as the recent HI image of Messier 101 (Figure 16). A comparison with the very early WSRT image shown in Figure 1 demonstrates the degree of improvement the WSRT has undergone since the opening in 1970.

The development of even more sophisticated software to enable proper and careful analysis of the neutral hydrogen data has also been very important during the past several decades. The Groningen Image Processing System (GIPSY, www.astro.rug.nl/ gipsy) still is a primary platform for the analysis of neutral hydrogen observations. It has been designed in the seventies and eighties with a keen eye for new software techniques incorporating novel ideas, in particular for image processing applications. It has been developed further over the years, has been transferred to new hardware and computing platforms and is now used by hundreds of astronomers around the world for the analysis of neutral hydrogen data from not only the Westerbork telescope but also from various similar instruments. 